\title{
Perfil das Egressas dos Cursos de Computação da UNIPAMPA: Uma Análise da Formação Acadêmica e da Atuação Profissional
}

\author{
Alice Fonseca Finger ${ }^{1}$, Andréa Sabedra Bordin ${ }^{1}$, Aline Vieira de Mello ${ }^{1}$ \\ ${ }^{1}$ Universidade Federal do Pampa (UNIPAMPA) \\ Av. Tiaraju, 810 - Ibirapuitã, Alegrete - RS, 97546-550 \\ \{alicefinger, andreabordin, alinemello\}@unipampa.edu.br
}

\begin{abstract}
This paper presents an analysis of academic training and professional experiences of female graduates of Computer Science (CS) and Software Engineering (SE) at UNIPAMPA. Data were collected through a questionnaire elaborated from several references. From complete answers, it was possible to define the profiles of the graduate of the undergraduate programs, as well as to point out the most significant similarities and differences. It was observed that graduate females from CS tend to pursue the academic master degree, while those from SE immediately look for work experiences.
\end{abstract}

Resumo. Este trabalho apresenta uma análise da formação acadêmica e atuação profissional das egressas dos cursos de Ciência da Computação (CC) $e$ Engenharia de Software (ES) da UNIPAMPA. Os dados foram coletados através de um questionário elaborado com base em diversas referências. A partir das respostas completas, foi possivel definir os perfis das egressas dos cursos, bem como pontuar as similaridades e diferenças mais significativas. Observou-se que egressas de CC tendem a realizar o mestrado acadêmico, enquanto as de ES se inserem imediatamente no mercado de trabalho.

\section{Introdução}

Historicamente, a área da computação é vista como predominantemente masculina, e isso se comprova quando observamos os números de ingressantes e concluintes dos cursos de graduação por gênero [SBC 2018]. Segundo Saboya [Saboya 2009], o mercado de trabalho nessa área também foi organizado a partir dos valores masculinos, que muitas vezes possuem concepções machistas que são agressivas a presença de mulheres. Esse cenário faz com que muitas mulheres não percebam a computação como um espaço de atuação e mesmo aquelas que fazem graduação na área demonstram insegurança em relação ao que vão enfrentar no mercado de trabalho.

Entender o processo de formação acadêmica e de escolhas profissionais de mulheres da área da Computação, ou seja, em quais projetos se envolveram, por quais áreas de pesquisa se interessaram, assim como onde estão, o que estão fazendo e de que forma a graduação impactou nas escolhas após graduadas, permite traçar um perfil dessas mulheres, o qual fornece importantes subsídios para as mais diversas tomadas de decisões, como apresentar a real inserção das egressas, incentivando a permanência das graduandas assim como o ingresso de mulheres nos cursos. 
Ao procurar pela palavra "perfil" nos anais do Women in Information Technology (WIT) foram encontrados 11 artigos. Destes, somente 2 artigos apresentam o perfil profissional na área da Computação. O artigo de Martimiano [Martimiano et al. 2018] traz o perfil das profissionais de Tecnologia da Informação (TI) na cidade de Maringá-PR, apresentando os cargos ocupados, a percepção sobre preconceitos de gênero e a percepção sobre competência. Já o artigo de Silva [da Silva et al. 2019] apresenta o perfil das egressas dos cursos de Computação da Universidade Federal da Paraíba - Campus IV, abordando o tipo de pós-graduação realizada, o cargo que ocupam no mercado de trabalho, bem como o número de empresas em que já atuaram e os cargos ocupados.

O presente trabalho traz informações sobre a formação acadêmica das egressas dos cursos de Ciência da Computação (CC) e Engenharia de Software (ES) da Universidade Federal do Pampa (UNIPAMPA) desde a graduação até a pós-graduação, a atuação no mercado de trabalho e a percepção sobre tratamento diferenciado em decorrência de gênero. Diferentemente dos trabalhos citados, também são abordados itens como a participação em projetos, a realização de estágio, assim como são realizadas diversas análises quantitativas e qualitativas. Com base nessas informações foi possível estabelecer o perfil de egressas de CC e ES, identificar suas similaridades e diferenças.

O restante deste trabalho está organizado como segue. A metodologia adotada, desde a elaboração do instrumento de coleta de dados até a análise dos resultados, é descrita na Seção 2. Na Seção 3 são apresentados os resultados, os quais são discutidos na Seção 4. Por fim, as considerações finais são apresentadas na seção 5.

\section{Metodologia}

A pesquisa foi realizada no contexto do Projeto Gurias na Computação [Ferrão et al. 2017], com egressas dos cursos Ciência da Computação (CC) e Engenharia de Software (ES) da UNIPAMPA, no período de fevereiro a abril de 2020. O instrumento utilizado para a coleta de dados foi um questionário elaborado a partir de várias referências bibliográficas e documentos técnicos, dentre eles [da Silva Figueiredo et al. 2018] e [Brasscom 2017]. O questionário contém entre 70 e 210 questões (dependendo das respostas fornecidas) divididas em três dimensões: sociodemográfica, formação acadêmica e atuação profissional.

A dimensão sociodemográfica contém as seguintes questões: sexo; data de nascimento; estado civil; número de filhos e data de nascimento de cada filho; cidade, estado e país de residência. A questão sobre sexo está presente porque, embora o foco deste artigo seja as egressas, o questionário foi pensado para ser usado no acompanhamento de egressos, independente de sexo.

A dimensão formação acadêmica contempla a graduação e pós-graduação. A parte referente à graduação possui questões sobre bolsas de estudo, estágio e trabalho durante a graduação, e o Trabalho de Conclusão de Curso (TCC). Já a parte referente à pós-graduação traz questões que abordam a realização de curso de especialização ou MBA, mestrado e doutorado. Adicionalmente, existem questões sobre as contribuições da graduação para a continuidade dos estudos e questões sobre tratamento diferenciado em decorrência de gênero.

A dimensão atuação profissional contém questões sobre a inserção no mercado de trabalho, o número de empresas em que atuou e para cada empresa, seu nome, cidade, 
estado, país, cargo ocupado, modalidade de contratação, faixa remuneração, entre outros. Adicionalmente, existem questões sobre as contribuições da graduação para a atuação profissional e questões tratamento diferenciado no mercado de trabalho.

O questionário foi desenvolvido utilizando a ferramenta LimeSurvey e pode ser acessado na íntegra através do link https://bit.ly/egressasunipampa. O questionário foi enviado por e-mail para as egressas, que também foram incentivadas a responder através de redes sociais e aplicativos de mensagens. O processamento das respostas foi realizado nas ferramentas de análise de dados RStudio e Anaconda Python. Foram feitas análises quantitativas e qualitativas e gerados gráficos de barras, pizza e boxplot.

\section{Resultados}

O questionário foi enviado para todas as egressas dos cursos Ciência da Computação (CC) e Engenharia de Software (ES), desde a criação dos cursos até o primeiro semestre de 2019, totalizando 25 egressas. Dessas, 16 responderam completamente o questionário, 4 responderam parcialmente e 4 não responderam. Os resultados apresentados nesta seção foram obtidos a partir das respostas completas, sendo 11 respostas de egressas graduadas em CC e 5 em ES. O número maior de respondentes do curso de $\mathrm{CC}$ é justificado porque esse curso foi criado 4 anos antes do curso de ES.

Sobre a dimensão sociodemográfica, a maioria das egressas (10) reside no estado em que a instituição do curso de graduação está situada, sendo 6 egressas na capital do estado e 4 na cidade em que a instituição está situada. As demais estão espalhadas pelos estados de São Paulo, Paraná, Distrito Federal e Santa Catarina. Todas as egressas tem menos de 34 anos, sendo a maioria solteira (10), 5 casadas e 1 divorciada. Somente uma egressa possui 1 filho.

\subsection{Formação Acadêmica}

\subsubsection{Graduação}

Com base nos dados do ano de ingresso e conclusão das egressas nos dois cursos de graduação (Figura 1), percebe-se que para ES, $50 \%$ das egressas que responderam o questionário integralizaram o curso no período de 5 a 7 anos, enquanto que na $\mathrm{CC}$ metade das egressas integralizou entre 4,5 e 5,5 anos. Ainda, destaca-se o fato de os dois cursos apresentarem 4 anos como tempo mínimo de integralização, sendo de 9 anos o tempo máximo para ES, enquanto na CC esse valor é de 7 anos. Cabe salientar que as duas amostras são de tamanhos distintos, logo não é possível relacionar os tempos de integralização.

A partir de uma análise descritiva do tempo de integralização do curso, tem-se que somente 4 egressas, do total de 16, conseguiram integralizar no período regular (4 anos), sendo 3 discentes de CC (aprox. 27\%) e 1 de ES (20\%). Em 5 anos, tem-se 5 egressas de CC (aprox. 45\%) e 1 de ES (20\%). As restantes, 3 de CC (27\%) e 3 de ES (60\%) levaram de 6 a 9 anos para integralizar. Mesmo que tenhamos uma amostra pequena de dados, fica possível identificar que grande parte das discentes da ES levou um tempo maior do que o regular para integralizar o curso. Já na CC, a grande maioria integralizou entre 4 e 5 anos.

Em busca de justificativas para um tempo de integralização diferente do regular, analisaram-se os dados referentes às 5 egressas que trabalharam durante a graduação. 


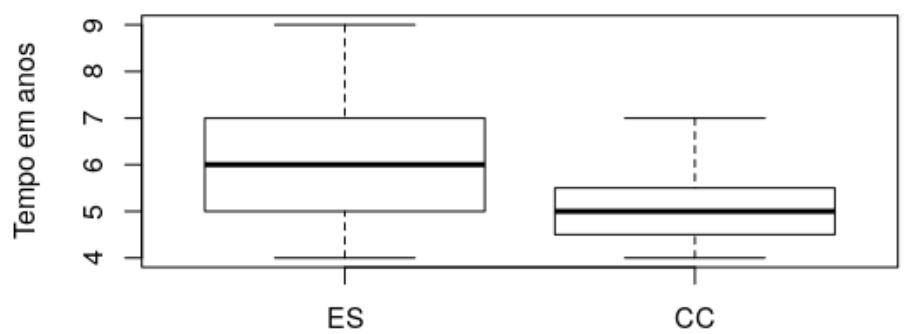

Figura 1. Tempos de integralização das egressas em cada curso

Das 4 egressas que concluíram o curso em tempo regular, apenas 1 trabalhou durante a graduação. Já dentre as 12 egressas que concluíram em 5 ou mais anos, apenas 4 trabalharam durante a graduação. Embora a amostra seja pequena, parece não existir uma relação direta entre o fato de trabalhar e o tempo de integralização do curso.

Todas as egressas de ES (5) realizaram estágio em instituições e setores de tecnologia de informação e comunicação, visto que o mesmo é obrigatório no curso. Neste caso, apenas 1 realizou o estágio em outra cidade e estado, sendo que as demais o realizaram na cidade onde a instituição de graduação está situada. Dentre as egressas de CC (11), apenas 3 realizaram estágio não-obrigatório, também na mesma área e cidade.

Dez egressas afirmaram ter sido bolsistas em projetos de ensino, pesquisa, extensão ou gestão, com atuação aproximada de sete meses em cada bolsa recebida, totalizando 24 bolsas. A distribuição dos tipos de bolsas por egressa é exibida na Figura 2, onde percebe-se um total de 7 bolsas de pesquisa, 6 de extensão e ensino e 5 de gestão, mostrando uma distribuição equilibrada em relação aos tipos de bolsas recebidas.

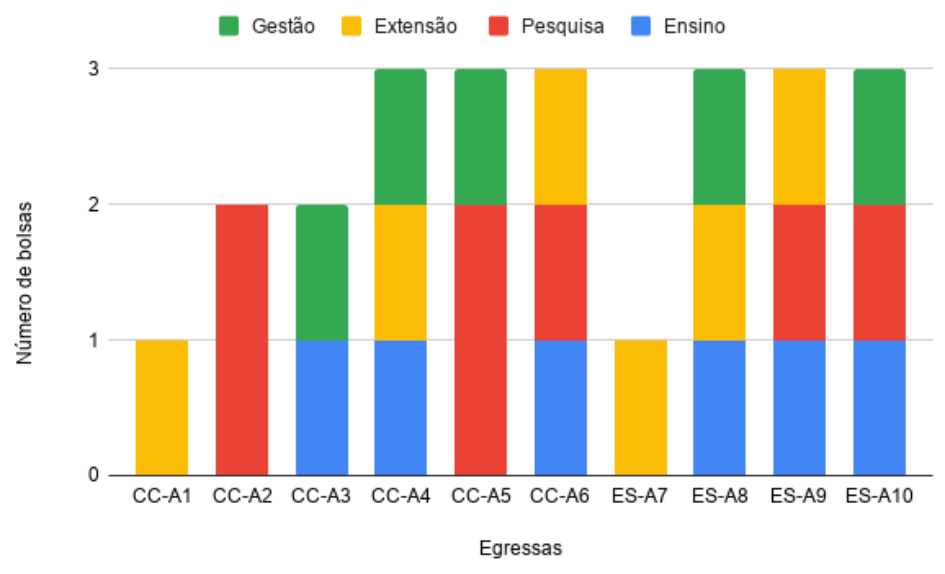

Figura 2. Tipos de bolsa de ensino, pesquisa, extensão e gestão por egressa

Sobre as áreas de pesquisa escolhidas para o desenvolvimento do TCC, observa-se na Figura 3 que tanto as egressas de CC quanto de ES optaram por áreas diversas. Cabe também destacar que entre as egressas de ES, 4 mencionaram a interdisciplinaridade de suas áreas de pesquisa com a área de Interação Humano-Computador (IHC), o que não é apresentado no gráfico. Com isso, percebe-se que há um interesse maior pela área de IHC 
em relação as demais áreas.

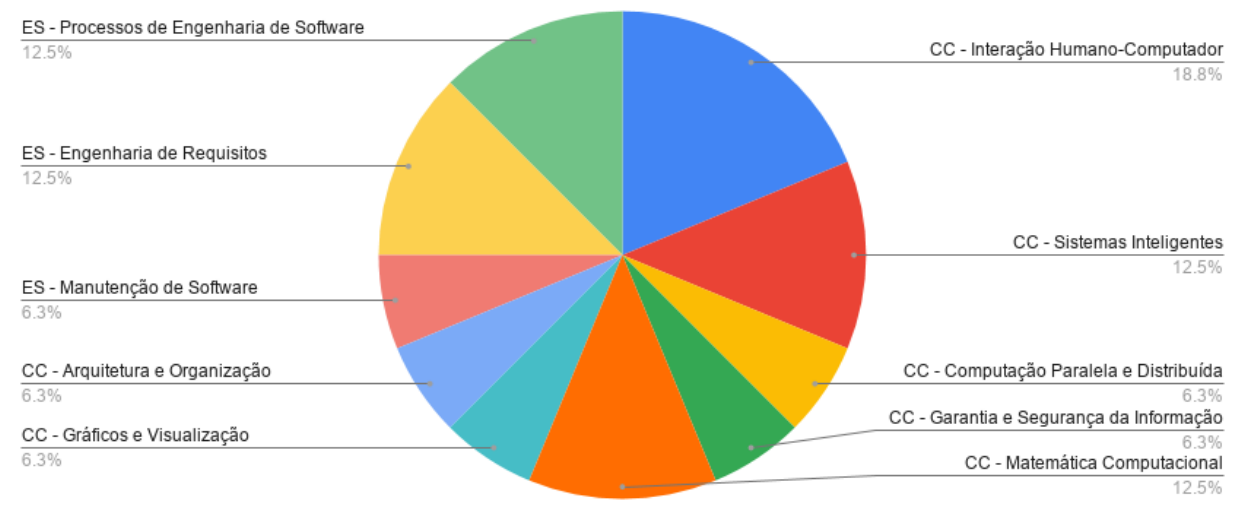

Figura 3. Número de egressas por áreas de pesquisa do TCC

Ao relacionar as áreas de pesquisa de TCC das egressas com as áreas das suas bolsas de pesquisa, observou-se que 3 egressas (de cinco egressas contempladas com 7 bolsas) desenvolveram TCC na mesma área das suas bolsas.

\subsubsection{Pós-Graduação}

Das 16 egressas respondentes do questionário, 11 continuaram a formação acadêmica após a conclusão da graduação, tanto em cursos de pós-graduação lato sensu como stricto sensu. Dessas, 5 fizeram cursos lato sensu, sendo 3 egressas do curso de ES e 2 do curso de CC. Três iniciaram a especialização no ano seguinte ao término da graduação em áreas da Computação diversas.

Oito egressas, incluindo 2 que fizeram especialização, optaram por fazer mestrado, todas de CC. Dessas, 7 iniciaram no ano seguinte à graduação. Duas egressas entraram em programas de pós-graduação em Engenharia Elétrica na mesma instituição onde se graduaram, 5 egressas em programas de pós-graduação em Ciência da Computação em instituições de todo o país e 1 egressa optou por um Mestrado Profissional em Informática na Educação. Duas egressas de CC já ingressaram no doutorado, uma no ano seguinte à conclusão do mestrado e a outra após dois anos. Ambas entraram em programas de pósgraduação em Ciência da Computação.

A Tabela 1 mostra as áreas de pesquisa das egressas que cursaram mestrado e doutorado, onde observa-se que não existe concentração em determinadas áreas. As áreas de pesquisa na graduação também estão presentes na referida tabela com a intenção de estabelecer uma possível relação de continuidade nas áreas. Analisando os TCC das egressas, observa-se que essa continuidade aconteceu nas pesquisas de graduação e mestrado de 5 egressas (CC2, CC4, CC5, CC7 e CC8). Assim, percebe-se que a escolha da área de pesquisa do TCC tende a se perpetuar no mestrado.

Com a finalidade de encontrar alguma relação entre egressas que foram bolsistas em projetos (ensino, pesquisa, extensão ou gestão) durante a graduação e optaram por continuar sua formação acadêmica, a Tabela 2 apresenta uma relação de frequências absolutas sobre duas variáveis: ser bolsista e fazer especialização, mestrado ou doutorado. 
Tabela 1. Áreas de pesquisa das egressas

\begin{tabular}{l|l|l|l} 
Egressas & Área TCC & Área Mestrado & Área Doutorado \\
\hline CC1 & Interação Humano-Computador & Visão Computacional & Interação Humano-Computador \\
CC2 & Sistemas Inteligentes & Inteligência Artificial & \\
CC3 & Computação Paralela e Distribuída & Modelagem e Otimização de Sistemas & Computação Paralela e Distribuída \\
CC4 & Garantia e Segurança da Informação & Redes e Comunicações & \\
CC5 & Gráficos e Visualização & Gráficos e Visualização & \\
CC6 & Matemática Computacional & Tecnologias Assistivas & \\
CC7 & Arquitetura e Organização & Arquitetura e Organização & \\
CC8 & Sistemas Inteligentes & Reconhecimento de Padrões & \\
\hline
\end{tabular}

Tabela 2. Frequência absoluta: ser bolsista e fazer pós-graduação

\begin{tabular}{c|c|c|c|c|c|c|c}
\multirow{2}{*}{ Bolsista } & Espc./Mest./Dout & \multicolumn{2}{|c|}{ Freq. abs. Esp } & \multicolumn{2}{|c}{ Freq. abs. Mest. } & \multicolumn{2}{|c}{ Freq. abs. Dout. } \\
\cline { 3 - 8 } & & CC & ES & CC & ES & CC & ES \\
\hline Não & Não & 5 & 0 & 2 & 1 & 4 & 1 \\
Sim & Não & 4 & 2 & 1 & 4 & 5 & 4 \\
Não & Sim & 0 & 1 & 3 & 0 & 1 & 0 \\
Sim & Sim & 2 & 2 & 5 & 0 & 1 & 0 \\
\hline Total & 11 & 5 & 11 & 5 & 11 & 5 \\
\hline
\end{tabular}

É possível perceber que das 11 egressas de CC que seguiram a formação acadêmica, 2 fizeram especialização, 8 foram para o mestrado e 2 optaram também pelo doutorado. Já na ES, de 5 egressas 3 fizeram especialização e nenhuma cursou mestrado ou doutorado. Especificamente em relação ao mestrado que possui o maior número de casos, das 8 discentes que cursam ou cursaram (todas de CC), 5 haviam sido bolsistas durante a graduação $(62,5 \%)$. Analisando a coluna de especialização, das 5 egressas (2 CC e 3 ES), 4 tiveram algum tipo de bolsa de projeto durante a graduação (80\%). Por fim, na coluna do doutorado, apenas 2 egressas de CC cursam ou cursaram, sendo que uma foi bolsista $(50 \%)$. A partir dessas análises podemos inferir que discentes que participam como bolsistas de algum projeto durante a graduação tendem a buscar uma formação acadêmica.

Para as 11 egressas que continuaram a formação acadêmica em cursos de especialização, mestrado e doutorado, a contribuição do curso na formação acadêmica foi classificada como ótima $(63,6 \%)$, boa $(27,3 \%)$ e excelente $(9,1 \%)$. As disciplinas que as egressas destacaram como relevantes, com mais frequência, na continuação da formação acadêmica são Estrutura de Dados II (6), Estrutura de Dados I (5), Algoritmos (5) e Linguagens Formais (3).

\subsection{Atuação Profissional}

Das 16 egressas que responderam ao questionário, metade considerou fácil e a outra metade difícil a inserção no mercado de trabalho. É importante destacar que 12 egressas já trabalharam ou trabalham. Das egressas que nunca trabalharam até o momento (4), metade considerou fácil a inserção, sendo que uma egressa justificou que, embora nunca tenha trabalhado, recebeu várias propostas de trabalho mas optou por realizar o mestrado.

Em relação ao tempo para o ingresso no mercado de trabalho, de 12 egressas, 4 levaram dois anos para ingressar no primeiro emprego. No entanto, 3 delas fizeram mestrado, sendo assim, apenas uma egressa demorou um pouco mais para se colocar. Os 
principais motivos facilitadores apontados pela egressas foram a participação em projetos; a escrita de artigos; a graduação em ES e a divulgação de vagas na universidade. Já os fatores dificultadores citados foram a falta de experiência, destacada por 3 egressas; a ausência de vagas na área de TI na cidade de realização do curso de graduação; e o fato de possuir o mestrado e, portanto, ser muito qualificada para a vaga.

A Figura 4 apresenta a remuneração das 12 egressas que já atuaram profissionalmente, considerando as diferentes experiências no mercado. É possível perceber que todas as egressas de CC atuaram em 1 ou 2 instituições, com exceção de uma egressa que atuou em 5. Já as egressas de ES tiveram entre 1 e 4 experiências profissionais. Cabe ressaltar que, embora as egressas de ES tenham em média menos tempo de graduadas, elas trocaram mais de instituição se comparadas às egressas de CC.

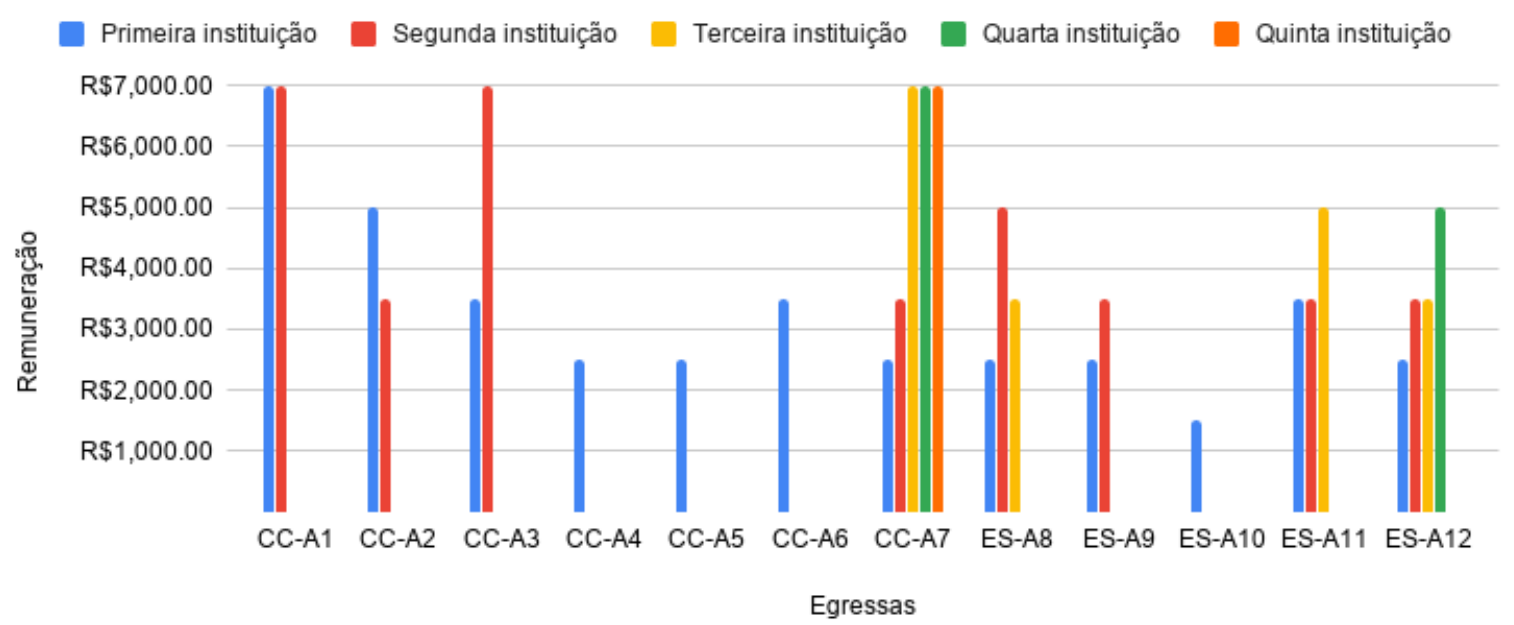

Figura 4. Remunerações das egressas nas empresas onde atuaram

Em relação às remunerações, a Figura 4 mostra que somente uma egressa entrou no mercado de trabalho ganhando mais de $\mathrm{R} \$ 5.000,00$, sendo que a média das remunerações nas instituições de ingresso no mercado de trabalho foram $\mathrm{R} \$ 3.700,00 \mathrm{e}$ $\mathrm{R} \$ 2.500,00$ para as egressas de CC e ES, respectivamente. Atualmente, somente 3 egressas estão na faixa de remuneração acima de $\mathrm{R} \$ 5.000,00$, sendo a média salarial atual das egressas de CC igual a $\mathrm{R} \$ 4.714,00$ e de ES igual a $\mathrm{R} \$ 3.700,00$. Observa-se, portanto, que as egressas de $\mathrm{CC}$ tendem a receber remunerações mais elevadas em relação às egressas de ES. No entanto, destaca-se que as egressas de CC possuem, em média, mais tempo no mercado de trabalho e com isso tiveram mais oportunidades de evolução salarial.

Ao todo, as egressas atuaram em 27 instituições localizadas majoritariamente no estado do Rio Grande do Sul (RS), seguido do estado do Paraná (PR). A grande maioria teve experiências na área de formação (11), atuando em instituições do ramo de Tecnologia da Informação (TI) ou Educacional. No ramo de TI, as egressas ocuparam, com mais frequência, os cargos de Analista de Desenvolvimento de Sistemas (4) e Analista de Processos de Negócios (2). As demais ocuparam cargos como: Gerente de Projetos de TI, Cientista de Dados, Scrum Master, Programador de Sistemas de Informação, Analista de Implantação e Analista de Qualidade. Já no ramo Educacional, todas as atuações foram no cargo de Professora. 
Sobre as disciplinas relevantes para o mercado de trabalho, as egressas de CC mencionaram com mais frequência as disciplinas de programação (4). Já as egressas de ES, destacaram as disciplinas de Análise de Software (2) e Processos de Software (2). Entre as egressas de ES, somente uma mencionou as disciplinas de Resolução de Problemas, conduzidas no curso através da metodologia ativa de Aprendizagem Baseada em Problemas, cujo objetivo é a preparação dos estudantes para o mercado de trabalho.

Por fim, quando indagadas sobre o nível de satisfação com a área, 7 egressas com experiência profissional se manifestaram. Algumas que trabalham no ramo da Educação, destacaram que a docência é muito desgastante e está cada vez mais desvalorizada pela sociedade, mas que ainda assim, permite autonomia em algumas decisões. Entre as que trabalham em empresas do ramo de TI, somente 1 se autodeclarou feliz, mesmo destacando os muitos desafios relacionados a pessoas no ambiente de trabalho. As demais mencionaram não se sentirem felizes em algum grau, relatando que faltava propósito, que não tinha sido possível trabalhar exatamente no que interessava e o desafio de ter que possuir várias softskills e ainda se manter atualizadas em relação a todas novas tecnologias que surgem.

\subsection{Percepção sobre tratamento diferenciado}

Das 16 egressas, 10 (62\%) indicaram ter recebido tratamento diferenciado tanto de colegas quanto de professores em decorrência de gênero durante sua formação acadêmica. Já um número ainda maior de egressas $(12-75 \%)$ indicaram ter percebido tratamento diferenciado dispensado a seus colegas. Nos comentários, as egressas apontaram que a maioria das pessoas subestima o potencial da mulher e o bom desempenho feminino muitas vezes é associado a plágio ou a beleza. Esses dados reforçam o quanto o ambiente universitário na área da Computação é machista.

Das 12 egressas que já atuaram no mercado de trabalho, 5 (41\%) afirmaram ter recebido tratamento diferenciado em seu ambiente de trabalho e 7 (58\%) indicaram já ter percebido algum tipo de tratamento diferenciado dispensado a seus colegas de trabalho. Nos comentários, as egressas apontaram que muitas vezes suas falas são ignoradas ou diminuídas; que elas normalmente não são incluídas em eventos sociais; e que perguntas sobre relacionamento e gravidez foram realizadas durante entrevistas de emprego.

\section{Discussão}

Com base nos resultados apresentados, buscou-se definir um perfil das egressas de CC e ES em relação às dimensões analisadas, de forma a identificar suas características, algumas similaridades e diferenças.

As egressas de CC necessitam em média 5,1 anos para integralizar o curso, a maioria não trabalha e nem realiza estágio durante a graduação. Mais da metade (55\%) foram bolsistas em projetos de ensino, pesquisa, extensão e/ou gestão. A maioria (73\%) continuou a formação acadêmica, realizando mestrado e, entre essas, 62,5\% foram bolsistas em algum projeto na graduação. A contribuição do curso de graduação na formação acadêmica foi considerada ótima ou excelente pela maioria (75\%), sendo as disciplinas de Estrutura de Dados e Algoritmos as mais relevantes. Um pouco mais da metade das egressas $(55 \%)$ afirmou ter recebido tratamento diferenciado em decorrência de gênero tanto de colegas quanto de professores durante a formação acadêmica. 
Quanto a atuação profissional, mais da metade da egressas de CC (64\%) informou ter tido alguma experiência profissional. A maioria (85\%) trabalhou em até 2 instituições dos ramos de TI ou Educação, localizadas majoritariamente no estado da instituição onde se graduaram. O salário médio atual dessas egressas é de $\mathrm{R} \$ 4.714,00$. As disciplinas de Programação foram as mais relevantes para as suas atuações profissionais. Metade das egressas $(50 \%)$ afirmou ter sofrido tratamento diferenciado em decorrência de gênero no mercado de trabalho.

Para integralizar o curso, as egressas de ES necessitam em média 6,2 anos e a maioria não trabalha durante a graduação. Em relação ao estágio, todas fazem, visto que é obrigatório para a conclusão do curso, e a grande maioria realiza na mesma cidade da instituição. Mais da metade (60\%) fez especialização e, dessas, $67 \%$ foi bolsista em projetos. A contribuição do curso na formação acadêmica dessas egressas foi considerada ótima pela maioria (67\%), sendo as disciplinas específicas de ES consideradas as mais relevantes. Um número expressivo das egressas (80\%) afirmou ter recebido tratamento diferenciado em decorrência de gênero tanto de colegas quanto de professores durante a formação acadêmica.

Todas egressas de ES tiveram alguma atuação profissional, sendo que a maioria trabalhou em pelo menos 3 instituições da área, mais frequentemente no ramo de TI e localizadas no mesmo estado da instituição de formação. $\mathrm{O}$ salário médio atual dessas egressas é de $\mathrm{R} \$ 3.700,00$. Foram destacadas as disciplinas de Análise de Software e Processos de Software como as mais relevantes para sua atuação profissional. Somente $40 \%$ das egressas afirmou ter recebido tratamento diferenciado no mercado de trabalho.

A partir dos dados acima, pode-se perceber algumas similaridades e diferenças em relação aos dois perfis de egressas. Como pontos em comum, observa-se a tendência das egressas que foram bolsistas de projetos em optar por fazer pós-graduação, além de não trabalharem enquanto alunas de graduação. Por fim, independente do curso, as egressas percebem um tratamento diferenciado em relação ao gênero na graduação, salientando que as egressas de ES o percebem com maior frequência.

Em relação às diferenças, no perfil específico de CC destaca-se a busca pela formação em cursos de mestrado, com uma tendência de seguir na área de pesquisa do TCC. Há uma média salarial maior e pouca oscilação em número de empresas trabalhadas. Já para ES, nota-se um tempo maior para integralização do curso e a opção pela inserção no mercado de trabalho, fato que pode ser justificado pelas alunas de ES cursarem estágio obrigatório durante a graduação. Egressas de ES em média apresentam uma faixa salarial menor e atuaram em um número maior de empresas.

\section{Considerações Finais}

Como contribuições principais desta pesquisa, destaca-se primeiramente, o instrumento de coleta de dados, elaborado através do esforço conjunto de um grupo de docentes da área de Computação da instituição, que utilizaram diversas referências para a elaboração do instrumento. As 70 questões estão distribuídas em várias dimensões de análise e permitem identificar um perfil completo, desde a formação acadêmica (graduação e pós-graduação) até o mercado de trabalho. Acredita-se que o instrumento possa servir de apoio ao desenvolvimento de pesquisas com o mesmo objetivo em outras instituições de ensino.

Na sequência, destaca-se que os resultados desta pesquisa trouxeram elementos 
que permitirão a criação de ações mais pontuais junto às graduandas, com objetivos diversos, tais como, divulgar às graduandas de ES as possibilidades de mestrado na sua área, assim como às graduandas de $\mathrm{CC}$, as possibilidades de atuação profissional; apresentar o mapeamento das empresas onde as egressas fizeram estágio ou trabalharam, uma vez que elas informaram quais recomendavam ou não; criar campanhas de conscientização para docentes e discentes para evitar os tratamentos diferenciados apontados, entre outros.

De uma maneira mais geral, os resultados podem servir para dirimir os receios que as alunas costumam sentir em relação à entrada no mercado de trabalho, assim como podem servir para incentivar as graduandas a permanecerem nos cursos, uma vez que mostra um cenário positivo, na medida em que a maioria está, ou inserida no mercado, ou continuando sua formação acadêmica.

Por fim, entende-se que uma das ameaças à validação desta pesquisa é o tamanho do instrumento, que pode ter desmotivado a participação, bem como influenciado na qualidade das respostas. Além disso, embora $64 \%$ do total de egressas tenha respondido completamente o instrumento, o tamanho da amostra (16) representa outra ameaça a pesquisa porque impacta na confiabilidade dos resultados obtidos.

\section{Referências}

Brasscom (2017). Guia de Funções de Tecnologia da Informação e Comunicação no Brasil. Brasscom. Disponível em: https://brasscom.org.br/wpcontent/uploads/2017/08/brasscom-guia_de_funcoes_de_tic_2a_edicao-2017.pdf.

Acesso em: 28 fev. 2020.

da Silva, J., Dantas, V., Figueiredo, R., Medeiros, S., and Costa, T. (2019). Perfil profissional das egressas dos cursos de Computação da Universidade Federal da ParaíbaCampus IV. In Anais do XIII Women in Information Technology, pages 79-88, Porto Alegre, RS, Brasil. SBC.

da Silva Figueiredo, K., de Azevedo, J. K. N., Azevedo, J. G., de Arruda dos Santos, K. A., de Souza Rosa Gomes, R., Ventura, T. M., and Maciel, C. (2018). Perfil dos Egressos e Egressas de Computação de Mato Grosso no Mercado de Trabalho. In Anais do IX Computer on the Beach, pages 209-306. Universidade do Vale do Itajaí.

Ferrão, I. G., de Mello, A. V., and Melo, A. M. (2017). Gurias na Computação: fortalecendo e incentivando a participação feminina no Ensino Superior. In Anais do XI Women in Information Technology. SBC.

Martimiano, L. A. F., de Lima, N. V., Feltrim, V. D., and Roder, L. (2018). Um estrato do perfil das profissionais de TIC na cidade de Maringá-PR. In Anais do XII Women in Information Technology, Porto Alegre, RS, Brasil. SBC.

Saboya, M. C. L. (2009). Alunas de engenharia elétrica e ciência da computação: estudar, inventar, resistir. $\mathrm{PhD}$ thesis, Universidade de São Paulo.

SBC (2018). Educação superior em computação Estatísticas - 2017. Sociedade Brasileira de Computação. Disponível em: https://www.sbc.org.br/documentosda-sbc/summary/133-estatisticas/1200-pdf-png-educacao-superior-em-computacaoestatisticas-2017. Acesso em: 28 fev. 2020. 